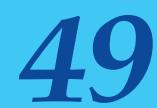

Anno XIV - gennaio 2018

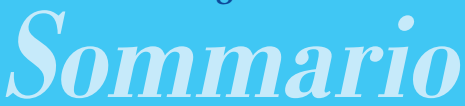

Editoriale

Per una riforma dei Ser.D. bisogna fare leva sugli operatori

Pietro Fausto D'Egidio

\section{SAGGI, STUDI E RICERCHE}

Orientare l'organizzazione dei Servizi verso un modello efficace e sicuro

di trattamento del dolore con farmaci oppiacei: I'esperienza della Casa

di Reclusione di Padova

Debora Lageder, Gaetano Morgillo, Livia Zuliani,

Giordano B. Padovan, Jessica Lorenzon,

Felice A. Nava

Carcere e clinica delle dipendenze.

Quali le sfide da affrontare per mantenere

terapeutico un sistema di cura?

Emanuele Bignamini, Sara Zazza,

Loredana Ierardi, Enrico Teta

"Lo Specchio Velato". La ricerca del Ser.D. di Orbassano sul fenomeno della complessità socio-sanitaria adolescenziale

Sabrina Anzillotti, Giuseppe Faro, Carola Morè

Position paper

Per un programma di eliminazione

della Epatite $C$ nella popolazione a rischio

dei consumatori di sostanze e dei detenuti

Felice A. Nava, Alfredo Alberti, Massimo Andreoni,

Sergio Babudieri, Giorgio Barbarini,

Pietro Fausto D'Egidio, Claudio Leonardi,

Alfio Lucchini

\section{CONTRIBUTI SCIENTIFICI}

Il fumo di "cannabis legale" come strumento per la riduzione del danno alla salute da tabacco

Fulvio Fantozzi

Il trattamento cognitivo-comportamentale di un caso di disturbo Borderline

della Personalità e Dipendenza

Martina Ceccarini

Sindrome di Steinert e dipendenza

da internet: valutazione e trattamento

di un caso clinico

Roberto Calabria, Antonella Sabatino,

Chiara Lirangi

\section{LE RUBRICHE}

Ad maiora

Procedura per la tenuta del fascicolo

personale dei pazienti

Raffaele Lovaste

Contaminazioni

Storie edificanti dall'altro mondo

Maurizio Fea

RECENSIONI

NOTIZIE IN BREVE

\section{FeDerSerD/FORMAZIONE}

Calendario 2018 - Eventi formativi FeDerSerD 68

\section{DOCUMENTO}

Carta dei Servizi "Doppia Diagnosi" (FeDerSerD-SIP-SINPIA)

\section{Mission \\ ITALIAN QUARTERLY JOURNAL OF ADDICTION}

\section{Per una riforma dei Ser.D. bisogna fare leva sugli operatori}

Dobbiamo cercare di fare uno sforzo per comprendere la realtà dei nostri servizi all'interno del SSN nel suo complesso.

La sanità è un grande bacino di impiego di lavoro qualificato a tutti i livelli. Il nuovo governo avrà il compito di elaborare un piano decennale dei fabbisogni di personale e di formazione, che incroci in maniera puntuale la domanda e l'offerta formativa.

La prima tappa di questo percorso sta nel superare le condizioni di precarietà del personale sanitario.

Alcuni temi sono particolarmente rilevanti: il coinvolgimento di noi operatori, la formazione, la assunzione dei nuovi operatori e la stabilizzazione dei precari.

\section{Coinvolgimento dei professionisti}

Noi operatori della sanità siamo i protagonisti di un paradigma della medicina moderna che è quello della complessità.

La clinica ci pone di fronte a pazienti in situazioni complesse che sono sanitarie e sociali, che coinvolgono molte primarie agenzie, e per le quali la società richiede risposte altrettanto complesse, attraverso una medicina che è fatta da una attività che ormai è solo e solamente interprofessionale e interdisciplinare.

Uno dei guai della medicina moderna è proprio che ci sono dei limiti alla nostra azione e ai nostri risultati; e che il falso senso di onnipotenza della idea "medicina" veicolata dai mass media crea illusioni e delusioni che poi si scontano anche sul piano sociale, sul piano antropologico e in effetti dovremmo riscoprire e capire che, intanto, fare il medico (e le altre professioni sanitarie e sociosanitarie) significa cercare di aiutare le persone.

- Studio sulla valutazione di esito dei corsi info-educativi svolti nella Regione Emilia-Romagna per i conducenti con infrazione dell'art. 186 e 186 bis del Codice della Strada "Guida in stato di ebbrezza" Claudio Annovi, Sara Querci, Giovanni Greco, Marilena Durante

- Una riflessione sull'efficacia delle politiche preventive per il controllo dei consumi di bevande alcoliche

Allaman Allamani, Franca Beccaria, Corrado Celata, Francesco Cipriani, Shlomo Stan Einstein, Pasquale Pepe, Franco Prina, Sara Rolando, Fabio Voller

- Consumo di alcol e tabacco negli studenti infermieri: uno studio osservazionale

Francesco Galli, Fabiana Piergigli, Carla Pierini, Stefano Fornaroli, Martina Cocchiglia, Dania Comparcini

Newsletter "Clinica dell'Alcolismo", Anno VII, n. 25 


\section{Mission}

Italian Quarterly Journal of Addiction

Periodico trimestrale della federazione italiana degli operatori dei dipartimenti e dei servizi delle dipendenze

\section{FrancoAngeli}

ANNO XIV, 2018 - N. 49

\section{Fe Der Ser D}

\section{Editor in Chief}

Alfio Lucchini, ASST Melegnano e Martesana - Milano Scientific Board

Roberta Balestra, ASUI Trieste; Claudio Barbaranelli, Università La Sapienza, Roma; Bruno Bertelli, Università di Trento; Stefano Canali, SISSA Trieste; Vincenzo Caretti, Università LUMSA, Roma; Ivan Cavicchi, Università La Sapienza e Tor Vergata, Roma; Massimo Clerici, Università Milano Bicocca: Massimo Diana, ASL Cagliari; Pietro Fausto D'Egidio, ASL Pesara; Ricar C. Gati, ASST Sant Ca lo Paolo Pescara, Riccardo C. Gatt, ASST Santi Carlo e Paol Mark D. Griffiths, Trent University di Nottingham Regno Unito; Jakob Jonsson, Spelinstitutet AB/Sustainable Interaction e Stockholm University Svezia; Enrico Molinari, Università Cattolica del Sacro Cuore Milano; Sabrina Molinaro, CNR Pisa; Felice Nava, ASL Padova; Marco Riglietta, ASST Papa Giovann XXIII Beramo; Ciorio Serio, ASP Palermo; Enrico Tempesta, Osservatorio Alcol e Giovani Roma

Editorial Board

Sandra Basti (Pavia); Guido Faillace (Trapani); Maurizio Fea (Pavia); Raffaele Lovaste (Trento); Finio Luchin (Bergam); Erio Ma Margherita Taddeo (Taranto); Franco Zuin (Milano); Concettina Varango (Lodi)

Editorial Office

Via Mazzini 54, 20060 Gessate (Mi),

tel. 3356612717

missiondirezione@tiscali.it

Proprietà: Fe Der Ser D

Sede legale

Via Giotto 3, 20144 Milano

Direttore responsabile: Stefano Angeli

Progetto grafico: Elena Pellegrini

Stampa: Geca Industrie Grafiche

Via Monferrato 54, 20098 San Giuliano Milanese

Lopera, comprese tutte le sue parti, è tutelata dalla legge sul diritto d'autore ed è pubblicata in versione digitale con licenza Creative Commons Attribuzione-Non CommercialeNon opere derivate 3.0 Italia (CC-BY-NC-ND 3.0 IT)

L'Utente nel momento in cui effettua il download dell'opera accetta tutte le condizioni della licenza d'uso dell'opera previste e comunicate sul sito http://creativecommons.org/licenses/by-nc-nd/3.0/it/legalcode

Copyright by FrancoAngeli s.r.l. - Milano

Poste Italiane s.p.a. - Sped. in Abb. Post. - D.L. $353 / 2003$ (conv. in L. $27 / 02 / 2004$ n. 46) art. 1 comma 1 - DCB Milano Autorizzazione Tribunale di Milano n. 131 del 6 marzo 2002

Chiuso in redazione il 31/01/2018

Edizione fuori commercio

Tiratura: 3.500 copie

ISSN 2037-4798

Seguici su:

www.facebook.com/FeDerSerd/

@FeDerSerd

(2) Gli articoli della rivista sono disponibili in modalità "Open Access" al link http://www.francoangeli.it/ riviste/sommario.asp?IDRivista=197\&lingua=it

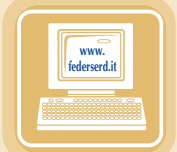

Non possiamo pensare di ottenere sempre il risultato della "guarigione", però in molti settori ci siamo illusi del fatto che invece non fosse così (pensate all'oncologia, a tanti miracolanti interventi chirurgici) e questo crea una situazione di sfalsamento per cui il richiamo di chi fa un lavoro come il nostro deve essere quello delI'umiltà; il compito del medico è quello di facilitare la resilienza ${ }^{1}$ e le strategie di coping 2 del paziente e questo è già un compito fondamentale, senza porsi limiti irraggiungibili.

\section{Quali azioni:}

- Gli operatori della sanità debbono poter portare il loro contributo, nell'interesse dei pazienti, in tutti i percorsi di organizzazione delle ASL attraverso la definizione di migliori procedure formalizzate.

- Premiare la qualità professionale e riconoscere il merito sia in termini economici che di carriera. Bisogna raggiungere questo obiettivo con procedure formalizzate che lascino meno spazi possibili a "forzature". È necessario che il vantaggio economico conseguito sia di entità significativa e per un tempo limitato.

\section{Formazione}

Nella sanità è necessario introdurre elementi che valorizzino la responsabilità individuale nell'impegno per una formazione continua.

\section{Quali azioni:}

La formazione in campo sanitario deve promuovere:

- L'aggiornamento costante sulle evidenze scientifiche "di base"; un aggiornamento su quelle conoscenze che debbono essere patrimonio comune di tutta una categoria professionale.

- L'aggiornamento costante sulle evidenze scientifiche "specialistiche"; un aggiornamento su quelle conoscenze che debbono essere patrimonio specifico in rapporto al ruolo professionale che si ricopre.

- L'aggiornamento costante sulle, e la partecipazione alla definizione delle, procedure di gestione della Azienda ASL e dello specifico settore di lavoro, la conoscenza delle normative di riferimento e le loro ricadute operative.

- Capacità nella costruzione e nel mantenimento di un clima relazionale positivo e costruttivo nel gruppo di lavoro, con il paziente e con le agenzie sociali di riferimento sul territorio.

\section{Nuove assunzioni}

Oggi la medicina ha bisogno di una coerenza tra la implementazione delle evidenze scientifiche e la numerosità e la qualità degli operatori sanitari.

È necessario fare un piano di assunzioni coerente con le necessità di una medicina moderna: una medicina capace non solo di applicare le straordinarie innovazioni tecnologiche ma anche di riscoprire il valore per la salute dei temi propri della prevenzione e della medicina sociale. Assistiamo ad un peggioramento epidemiologico, quest'ultimo imputabile al crescente invecchiamento della popolazione e al conseguente incremento delle patologie croniche.

Nel SSN italiano (rapporto OASI 2017, MIUR), il personale infermieristico è meno della metà rispetto alla Germania: 6 infermieri ogni 1000 abitanti contro 13.

Contemporaneamente, il 52\% dei medici ha più di 55 anni; si contano il doppio dei candidati alle specialità mediche rispetto ai contratti finanziati (13.802 versus 6.725). Se fosse vera l'ipotesi della persistente debolezza finanziaria, a fronte dell'aumento della cronicità e della LTC (residenzialità assistenziale a lungo termine), non potremmo che aumentare I'incidenza delle professioni sanitarie e degli operatori sociosanitari, sapendo che il costo medio aziendale di un medico equivale almeno a quello di due infermieri.

Questo impone però di modificare le competenze e i ruoli professionali.

Tra i medici occorre riflettere sulla quota di generalisti rispetto ai professionisti sempre più specializzati.

Nel mondo delle professioni sanitarie occorre distinguere tra crescita verticale nel coordinamento di pari, specializzazione nel contenuto tecnico professionale e sviluppo di ruoli contendibili con altri profili professionali: case manager, gestore di piastra, operations manager (rapporto OASI 2017, cap. 11 e ARAN, 2017, Retribuzioni medie nella pubblica amministrazione). 
Nello specifico della prevenzione e cura delle dipendenze osserviamo che in Italia ci sono 580 Ser.D. che, con circa 7200 operatori, curano ogni anno circa 300.000 pazienti.

La legge sui LEA (DPCM 12 gennaio 2017, pubblicato il 18 marzo in Gazzetta Ufficiale - Supplemento n. 15) riscrive e amplia il mandato istituzionale dei SerD e delle comunità.

Ma non sono state stanziate, né dal governo, né dalle regioni, le risorse minime necessarie per implementare le attività nel settore delle dipendenze così come previsto dagli artt. 28 e 35.

Se non assumiamo il personale nella misura necessaria:

- molti pazienti continueranno a restare senza cura;

- a molti pazienti non saremo in grado di offrire le tipologie di intervento necessarie;

- lo Stato continuerà a spendere molto danaro che altrimenti risparmierebbe, sui capitoli della Sicurezza Pubblica, della Amministrazione della Giustizia e della cura delle patologie correlate.

\section{Quali azioni:}

Impegnarsi a definire entro 3 mesi dalla assunzione di responsabilità di governo il numero di operatori da assumere per garantire i nuovi LEA.

La riorganizzazione complessiva dei servizi per la cura e la prevenzione delle dipendenze nelle sanità regionali non può e non deve essere guidata solo da una attenzione agli equilibri contabili della sanità.

Assumere nei Ser.D. il personale nella misura necessaria, che oggi viene stimata dalle società scientifiche del settore in nuove 6.000 unità.

\section{Stabilizzazioni}

Da oltre 10 anni si assiste ad una progressiva riduzione delle unità di personale nelle ASL a cui si cerca di sopperire con lavoro precario, interinale, financo con borse di studio.
Con risultati invero molto modesti sia in termini di fabbisogno che di qualità che viene compromessa dalla estrema precarietà. Questo accade in una epoca storica in cui, ancor più, la medicina ha bisogno di una coerenza tra la implementazione delle evidenze scientifiche e la numerosità e la qualità degli operatori sanitari.

\section{Quali azioni: \\ È necessario sanare subito tutti i precari nelle ASL.}

Pietro Fausto D'Egidio Presidente FeDerSerD

\section{Note}

1. In psicologia, la resilienza è una parola che indica la capacità di far fronte in maniera positiva a eventi traumatici, di riorganizzare positivamente la propria vita dinanzi alle difficoltà, di ricostruirsi restando sensibili alle opportunità positive che la vita offre, senza alienare la propria identità.

Sono persone resilienti quelle che, immerse in circostanze avverse, riescono, nonostante tutto e talvolta contro ogni previsione, a fronteggiare efficacemente le contrarietà, a dare nuovo slancio alla propria esistenza e perfino a raggiungere mete importanti.

2. In psicoterapia cognitiva e psichiatria il termine coping (termine inglese traducibile con "strategia di adattamento") indica l'insieme dei meccanismi psicologici adattativi messi in atto da un individuo per fronteggiare le situazioni potenzialmente stressanti o pericolose per il normale funzionamento psichico e il normale stato di benessere psicofisico. Attraverso l'insight l'individuo prende consapevolezza delle proprie vulnerabilità e limiti nelle situazioni varie del vivere quotidiano e di conseguenza cerca di sviluppare e mettere in atto il coping nella maniera più efficace possibile. Tali livelli di consapevolezza e gestione sono in genere normalmente sviluppati nel tempo oppure sotto la guida di uno psicoterapeuta nelle sedute psicoterapeutiche.

\section{RECENSIONE}

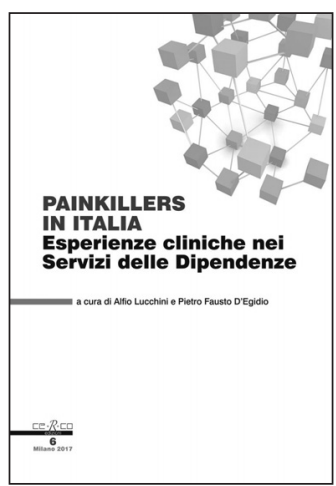

Alfio Lucchini, Pietro Fausto D'Egidio (a cura di)

\section{PAINKILLERS IN ITALIA \\ Esperienze cliniche nei Servizi delle Dipendenze}

pp. $152, € 30,00$

Editore: Cerco Edizioni

È importante che inizi anche nel nostro Paese una riflessione sul fenomeno dei Pain Killers che non sia solo aneddotica o un semplice richiamo alla giusta attenzione per il sistema sanitario basandoci sulla realtà preoccupante degli Stati Uniti.

L'apprendere che negli USA la mortalità acuta indotta da pain killers ha superato di 5 volte quella da eroina favorisce una prima riflessione.

La situazione italiana è assai differente: la prescrizione di oppioidi da parte dei medici di medicina generale è ancora lontana dal raggiungimento di quegli standard che possono garantire la gestione efficace del dolore, oltre al fatto che esistono sistemi in atto che pre- vengono la iperprescrizione; e poi esiste un sistema di intervento sulle dipendenze diffuso su tutto il territorio italiano - i Ser.D. che potrebbe garantire una risposta adeguata.

FeDerSerD, nel 2016 ha proposto un premio nazionale (il XII) per la presentazione di lavori scientifici scegliendo tre temi: la epidemiologia a livello dei territori della dipendenza da oppioidi prescritti; le esperienze di trattamento della dipendenza da oppioidi prescritti nella terapia del dolore; la organizzazione dei servizi con modelli orientati al trattamento di questi pazienti.

I lavori scientifici vengono qui presentati e propongono approfondimenti di ottimo livello, dei Ser.D. con l'apporto anche di servizi ospedalieri ed universitari. Per i servizi delle dipendenze una nuova sfida per salvaguardare la salute in particolare dei nostri giovani, per FeDerSerD il dovere di approfondire lo sviluppo di questo fenomeno e di essere accanto ai Servizi e ai professionisti tutti interessati per gli aspetti conoscitivi, formativi, organizzativi che affrontare un settore così complesso richiede.

Alfio Lucchini, medico, psichiatra, specialista in psicologia, psicoterapeuta, direttore del dipartimento salute mentale e delle dipendenze ASST Melegnano e della Martesana (Milano). Responsabile del settore "Terapia del dolore severo e dipendenze" di FeDerSerD.

Pietro Fausto D'Egidio, medico, specialista in medicina interna, ematologia generale, allergologia e immunologia clinica. Già direttore del Ser.D. di Pescara è presidente nazionale di FeDerSerD. 